\title{
Antioxidants and Mineral Contents of Chicory as Coffee Additive
}

\author{
Uğur Başarann ${ }^{1, a}$, Erdem Gülümser ${ }^{2, b, *}$, Cennet Yaman ${ }^{1, c}$, Medine Çopur Doğrusöz ${ }^{1, d}$, Hanife Mut ${ }^{2, e}$ \\ ${ }^{1}$ Department of Field Crops, Faculty of Agriculture, Bozok University, 66200 Yozgat, Turkey \\ ${ }^{2}$ Department of Field Crops, Faculty of Agriculture and Natural Science, Bilecik Şeyh Edebali University, 11230 Bilecik, Turkey \\ *Corresponding author

A R T I C L I N F O A B S T R A C T \\ Research Article \\ In this study, roots of Turkish origin wild chicory (Cichorium intybus L.) genotypes were \\ investigated for total flavonoid and phenolic contents, radical cation scavenging activity (ABTS), \\ Free radical scavenging activity (DPPH), and radical, mineral content. These characteristics were \\ also compared with other coffee varieties. The total flavonoid and phenolic contents ranged between \\ 0.290-4.350 mg QE/g dry weight (DW) and 0.943-13.860 mg GAE/g DW. The DPPH was listed \\ Received : 02/09/2020 \\ Accepted : 19/10/2020 \\ here from high to low value: raw coffee beans $=$ roasted coffee beans $>$ roasted fruits of turpentine \\ tree $>$ instant coffee $=$ roots of chicory. The content of $\mathrm{P}, \mathrm{Ca}, \mathrm{Mg}, \mathrm{Zn}, \mathrm{B}, \mathrm{Cr}$, Co and Mo ranged \\ between $0.71-2.78 \%, 0.25-0.46 \%, 7.29-20.66,4.44-11.07,0.40-1.67,0.49-5.48$ and 5.69-14.46 \\ ppm, respectively. As a result, chicory roots exhibited low antioxidant activity, but higher mineral \\ Keywords: \\ Antioxidant \\ content compared to the other tested coffee varieties which indicates that chicory could be used a \\ coffee additive.
}

Chicory

Coffee additive

Mineral content

Root

\section{Introduction}

Cichorium sp. genus is widely distributed in Africa, Asia, Europe, Australia, Northern America, and Southern America. Cichorium intybus L. and Cichorium endivia L. are mainly cultivated. $C$. intybus differs from $C$. endivia in terms of short capsules and persistency. $C$. intybus is common species compared to $C$. endivia. C. intybus is extremely tolerant to high temperature and drought due to its capability of elongation, and protecting its greenery during the summer and capability of growing without being watered in marginal areas (Kiers et al., 1999).

C. intybus is cultivated as a medicinal plant. Judzentienne and Udien (2008) reported that chicory roots contain $40 \%$ inulin. Its compounds such as, bitter sesquiterpene lactones, coumarins, flavonoids and vitamins are also used as anti-hepatotoxic, antiinflammatory, liver tonic, cholagogue, depurative, diuretic, emmenagogue, alexeteric, and used as tonic, anticancer and other medicinal purposes (Franck, 2002; Nandagopal and Ranjitha 2007; Denev et al., 2012).
Previous studies showed that chicory roots have constituents such as caffe-oylquinic acid, quercedina, antidiabetic, antihepatotoxic, anti-inflammatory and antioxidant activities (Ahmad et al., 2002; Schumacher et al., 2011; Ghamarian et al., 2012; Jurgonski et al., 2012; Kaskos, 2012). On the other hand, chicory roots have been considered as coffee additives since ancient times. Chicory coffee which is caffeine-free is the source of plant phenolics, and phenolic content is correlated with antioxidant activity (Lavelli, 2008). Chicory coffee is a source of caffeic acid and a healthy person is recommended to consume $300 \mathrm{ml}$ chicory coffee per week (Schumacher et al., 2011). Besides, the plant has anti-inflammatory, antiviral and anti-cancer characteristics.

The coffee contains significant nutrients vital for energy and health, but the biggest problem in coffee is additives. These substances are not organic and create health risk (Yiteyal and Tilahun, 2017). Therefore, there is a need for additives of plant origin. Chicory is used as 
coffee additives, preservatives, and digestive stimulant action. The aim of this study is to determine the antioxidant composition and mineral contents of wild chicory roots collected from 27 different locations in Turkey, and to compare with commercial or traditional coffee varieties (roasted fruits of turpentine tree, instant coffee, roasted coffee beans, and raw coffee beans).

\section{Material and Methods}

In this study, roots of $C$. intybus "Chicory" genotypes, roasted fruits of turpentine tree (Pistacia terebinthus L.), instant coffee, roasted coffee beans and raw coffee beans were used. (The coffee varieties used as control were provided from markets). The roots of chicory genotypes were obtained from the field experiments in Yozgat/Turkey. Initially, chicory seeds were collected from 27 different locations of Turkey (Table 1) in 2014.

Seeds were sown in peat media and were transferred to the field with 50x50 m of dimension distance in May 2015 in Yozgat/Turkey, and roots were harvested at the end of October, the same year. The experimental area soil gathered from $0-30 \mathrm{~cm}$ deep included $\mathrm{pH}$ of $8.20,7.93 \%$ $\mathrm{CaCO} 3,86.2 \mathrm{~kg} \mathrm{ha}^{-1}$ phosphorus, $484.7 \mathrm{~kg} \mathrm{ha}^{-1}$ potassium, and $1.91 \%$ organic matter. Mean, long term annual precipitation of Yozgat is about $574.4 \mathrm{~mm}$ and the mean temperature is $9.0{ }^{\circ} \mathrm{C}$. Mean rainfall of Yozgat throughout $2015(717.1 \mathrm{~mm})$ was higher than the precipitation means for a long time. Mean temperature in growing season in 2015 was $10.0^{\circ} \mathrm{C}$ (Anonoymous, 2019).

After the harvesting, the roots were cleaned and roasted at $140{ }^{\circ} \mathrm{C}$ temperature for 2 hours, then crushed using a conventional method. Roasted fruits of turpentine tree $(P$. terebinthus), instant coffee, roasted coffee beans and raw coffee beans were provided from commercial coffee suppliers and analyzed for same traits.

Harvested, dried and finely ground samples (weighing about $5 \mathrm{~g}$ per plant) of chicory roots, roasted fruits of turpentine tree, instant coffee, raw and roasted coffee beans were extracted in methanol at $40{ }^{\circ} \mathrm{C}$ for $24 \mathrm{~h}$. The mixtures were filtered through Whatman paper and, methanol was separated with a rotary evaporator (Heidolph, laborota 4000) to obtain extract yields. Then, extracts were dissolved in methanol. Besides, different ratio of the roasted coffee bean and chicory root extracts (1:4, 2:3, 3:2, $4: 1)$ were prepared as total $1000 \mu \mathrm{g} \mathrm{mL}^{-1}$ in methanol for synergistic activity.

The total phenolic contents of samples were determined with slight modification according to the Folin-Ciocalteu reagent (FCR) method of Singleton et al. (1999). Samples $(200 \mu \mathrm{L})$ were mixed with diluted FCR $(200 \mu \mathrm{L})$ and shaken vigorously for $3 \mathrm{~min}$. Then, $600 \mu \mathrm{L}$ Sodium Carbonate $\left(\mathrm{Na}_{2} \mathrm{CO}_{3}\right)$ solutions $(20 \%)$ were added, and absorbance of each sample was measured at $760 \mathrm{~nm}$ after incubating in dark at room temperature for $2 \mathrm{~h}$. The total phenolic contents were expressed as mg equivalents of Gallic acid (GAE) $\mathrm{g}^{-1}$ dry weight (DW) according to the equation obtained from the standard Gallic acid graph and calculated from the calibration curve $\left(R^{2}=0.9994\right)$.

Total flavonoid content of each sample was determined with a method which was partially modified and adopted by Arvouet-Grand et al. (1994). Briefly, each sample (500 $\mu \mathrm{L})$ were mixed with $100 \mu \mathrm{L}$ of aluminum nitrate $(10 \%)$ and $100 \mu \mathrm{L}$ of potassium acetate $(1 \mathrm{M})$. Total volume of the solution was adjusted to $5 \mathrm{~mL}$ with ethanol. Similarly, a blank was prepared by adding methanol in place of sample. Absorbance measurements were read at $417 \mathrm{~nm}$ after $40 \mathrm{~min}$ incubation at room temperature in dark conditions. Total flavonoid content was expressed as $\mathrm{mg}$ equivalents of quercetin (QE) $\mathrm{g}^{-1} \mathrm{DW}$ according to the equation obtained from the standard quercetin graph and calculated from the calibration curve $\left(\mathrm{R}^{2}=0.9994\right)$.

The effect of each sample on 2.2-diphenyl-1-picrylhydrazyl-hydrate (DPPH) radical was identified according to Gezer et al. (2006). Two hundred microliter from each sample in methanol was added to $3.2 \mathrm{~mL}$ of $0.004 \%$ methanol solution of DPPH. Absorbance of each sample was read at 517 $\mathrm{nm}$ after $30 \mathrm{~min}$ incubation at room temperature in dark.

ABTS radical cation scavenging activity was estimated according to Miller et al. (1993) and Re et al. (1999) with a partial modification. $\mathrm{ABTS}^{+}$radical cation was obtained directly by reaction $30 \mathrm{mg}$ ABTS with $6.6 \mathrm{mg}$ potassium persulfate in $7.8 \mathrm{~mL}$ double-distilled water, and allowing the mixture to remain for $12-16 \mathrm{~h}$ in dark at the room temperature. Then, ABTS solution was diluted with bi-distilled water to an absorbance of $0.700 \pm 0.020$ at $734 \mathrm{~nm}$. Each sample in methanol $\left(100 \mu \mathrm{L}\right.$ from $\left.1000 \mu \mathrm{g} \mathrm{mL}^{-1}\right)$ was added to ABTS solution $(2.8 \mathrm{~mL})$ and mixed. A blank was prepared by adding methanol instead of sample solution. Absorbance of each sample was read at $734 \mathrm{~nm}$ after at $30 \mathrm{~min}$ incubation at room temperature.

One-gram powdered samples were burned at $550{ }^{\circ} \mathrm{C}$ then $4 \mathrm{ml} 3 \mathrm{~N} \mathrm{HCL}$ added and the researchers waited 30 minutes for the ash to subside. Ash solution was filtered and pure water was added until the solution became $50 \mathrm{ml}$. Phosphorus (P), calcium $(\mathrm{Ca})$, magnesium $(\mathrm{Mg})$, zinc $(\mathrm{Zn})$, iron $(\mathrm{Fe})$, manganese $(\mathrm{Mn})$, sulphur $(\mathrm{S})$, boron $(\mathrm{B})$, chromium $(\mathrm{Cr})$, cobalt (Co) and molybdenum (Mo) concentrations in chicory root. Coffees (roasted fruits of turpentine tree, instant coffee, roasted coffee beans and raw coffee beans) were determined by inductively coupled plasma mass spectrometry (ICP-MS) using a Thermo Scientific- iCAPQc (Bremen, Germany).

The data were analyzed using the statistical package SPSS 16.0 V. Probabilities less than 0.05 were considered significant. Duncan's multiple range tests was used to separate the treatment means. All significant main effects were considered.

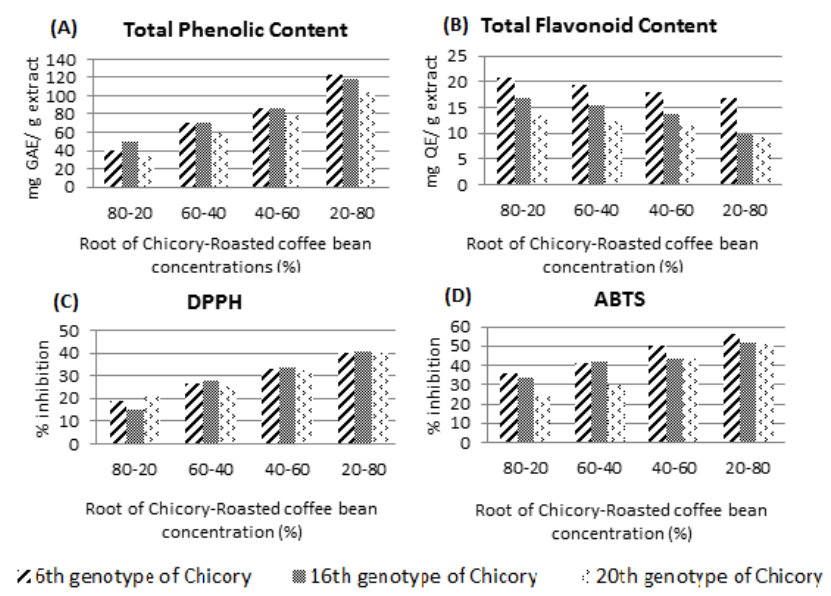

Figure 1. Total phenolic content (A), total flavonoid content (B), DPPH (C), ABTS (D) of mixtures ( $1 \mathrm{mg} / \mathrm{mL})$ belong to variation ratio of roasted coffee bean and root of $C$. intybus $\left(6^{\text {th }}, 16^{\text {th }}\right.$ and $20^{\text {th }}$ genotype of Chicory) 


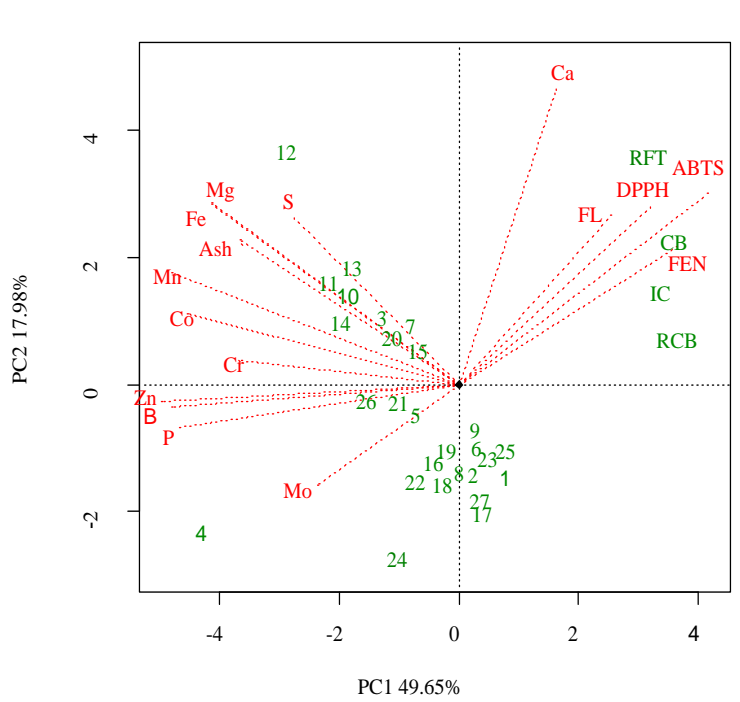

Figure 2. Principal component analysis of antioxidant properties and mineral contents of chicory roots and coffee varieties. 1-27, roots of chicory plants (Cichorium intybus) collected from different localities; RFT, roasted fruits of turpentine tree (Pistacia terebinthus); IC, instant coffee;

$\mathrm{RCB}$, roasted coffee beans; $\mathrm{CB}$, raw coffee beans.

\section{Result and Discussion}

Total bioactive components in chicory roots (RCP), roasted fruits of turpentine tree (P. terebinthus) (RFT), instant coffee (IC), raw coffee beans (CB) and roasted coffee beans (RCB) showed the presence of phenolics and flavonoids, and results were compared with each other.

Total flavonoid content of root extracts of chicory varied from $0.300 \mathrm{mg}$ to $1.146 \mathrm{mg} \mathrm{QE} / \mathrm{g} \mathrm{DW}$, while total phenolic contents varied from $0.943 \mathrm{mg}$ to $3.363 \mathrm{mg} \mathrm{GAE} \mathrm{g}^{-1} \mathrm{DW}$ (Table 1). It was also observed that there are significant differences among localities. The statistically highest total phenolic and flavonoid content in root extracts of chicory was emerged from extracts of the $15^{\text {th }}$ and the $13^{\text {rd }}$ genotypes (3.363 $\mathrm{mg} \mathrm{GAE} \mathrm{g}^{-1} \mathrm{DW}-1.120 \mathrm{mg} \mathrm{QE} \mathrm{g}^{-1} \mathrm{DW}$ and $3.533 \mathrm{mg}$ GAE g $\left.{ }^{-1} \mathrm{DW}-1.063 \mathrm{QE} \mathrm{g}^{-1} \mathrm{DW}\right)$. The extract of the $18^{\text {th }}$ genotype (0.943 $\mathrm{mg} \mathrm{QE} \mathrm{g}^{-1} \mathrm{DW}-0.296 \mathrm{mg} \mathrm{QE} \mathrm{g}^{-1} \mathrm{DW}$ ) was lowest.

Chicory has rich polyphenols content (Heimler et al., 2009), therefore the total phenolic content in the roots of chicory was higher than the total flavonoids. Besides, coffee varieties compared and the highest total phenolic content was found out to be instant coffee (13.860 mg GAE g-1 DW), while the lowest was roasted coffee beans (4.433 GAE g-1 DW). It was reported that instant coffee contains $4 \mathrm{mg} / \mathrm{g}$ of chlorogenic acid as nitrosatable compound, and also other phenolic compounds such as catechol, caffeic acid (Duarte et al., 2000).

Table 1. Origin of the chicory genotypes under investigation.

\begin{tabular}{|c|c|c|c|}
\hline Genotypes & Location & Latitude & Longitude \\
\hline 1 & Nevşehir-Avanos & $38^{\circ} 42^{\prime} 33.81^{\prime \prime} \mathrm{N}$ & $34^{\circ} 50^{\prime} 50.54^{\prime \prime} \mathrm{E}$ \\
\hline 2 & Yozgat-Yerköy & $39^{\circ} 39^{\prime} 10.7028^{\prime \prime} \mathrm{N}$ & $34^{\circ} 29^{\prime} 15.13^{\prime \prime} \mathrm{E}$ \\
\hline 3 & Samsun Central & $41^{\circ} 50^{\prime} 27.39^{\prime \prime} \mathrm{N}$ & $36^{\circ} 06^{\prime} 43.98^{\prime \prime} \mathrm{E}$ \\
\hline 4 & Konya-Meram & $37^{\circ} 40^{\prime} 24.89^{\prime \prime} \mathrm{N}$ & $32^{\circ} 28^{\prime} 08.23^{\prime \prime} \mathrm{E}$ \\
\hline 5 & Yozgat-Boğazliyan & $39^{\circ} 24^{\prime} 20^{\prime \prime} \mathrm{N}$ & $35^{\circ} 0^{\prime} 24^{\prime \prime} \mathrm{E}$ \\
\hline 6 & Yozgatl1-Central & $39^{\circ} 50^{\prime} 24.89^{\prime \prime} \mathrm{N}$ & $34^{\circ} 51^{\prime} 58.77^{\prime \prime} \mathrm{E}$ \\
\hline 7 & Yozgat-Sarıkaya & $39^{\circ} 32^{\prime} 22.12^{\prime \prime} \mathrm{N}$ & $35^{\circ} 15^{\prime} 15.70^{\prime \prime} \mathrm{E}$ \\
\hline 8 & Yozgat-Çandır & $39^{\circ} 14^{\prime} 36.15^{\prime \prime} \mathrm{N}$ & $35^{\circ} 31^{\prime} 01.91^{\prime \prime} \mathrm{E}$ \\
\hline 9 & Konya-Kulu & $39^{\circ} 05^{\prime} 20.98^{\prime \prime} \mathrm{N}$ & $33^{\circ} 02^{\prime} 40.04^{\prime \prime} \mathrm{E}$ \\
\hline 10 & Yozgat-Yerköy & $39^{\circ} 38^{\prime} 53.82^{\prime \prime} \mathrm{N}$ & $34^{\circ} 33^{\prime} 14.20^{\prime \prime} \mathrm{E}$ \\
\hline 11 & Yozgat-Yerköy & $39^{\circ} 38^{\prime} 20.45^{\prime \prime} \mathrm{N}$ & $34^{\circ} 27^{\prime} 48.98^{\prime \prime} \mathrm{E}$ \\
\hline 12 & Yozgat-Central & $39^{\circ} 49^{\prime} 54.33^{\prime \prime} \mathrm{N}$ & $34^{\circ} 48^{\prime} 32.43^{\prime \prime} \mathrm{E}$ \\
\hline 13 & Yozgat-Sorgun & $39^{\circ} 54^{\prime} 08.78^{\prime \prime} \mathrm{N}$ & $35^{\circ} 02^{\prime} 12.17^{\prime \prime} \mathrm{E}$ \\
\hline 14 & Yozgat-Sarıkaya & $39^{\circ} 34^{\prime} 54.63^{\prime \prime} \mathrm{N}$ & $35^{\circ} 25^{\prime} 43.97^{\prime \prime} \mathrm{E}$ \\
\hline 15 & Yozgat-Kadışehri & $39^{\circ} 57^{\prime} 22.28^{\prime \prime} \mathrm{N}$ & $35^{\circ} 39^{\prime} 52.68^{\prime \prime} \mathrm{E}$ \\
\hline 16 & Yozgat-Yerköy & $39^{\circ} 40^{\prime} 57.65^{\prime \prime} \mathrm{N}$ & $34^{\circ} 35^{\prime} 25.75^{\prime \prime} \mathrm{E}$ \\
\hline 17 & Yozgat-Boğazliyan & $39^{\circ} 8^{\prime} 19.30 .20^{\prime \prime} \mathrm{N}$ & $35^{\circ} 22^{\prime} 41.22^{\prime \prime} \mathrm{E}$ \\
\hline 18 & Kırşehir-Kaman & $39^{\circ} 21^{\prime} 24.93^{\prime \prime} \mathrm{N}$ & $33^{\circ} 41^{\prime} 51.41^{\prime \prime} \mathrm{E}$ \\
\hline 19 & Yozgat-Sarıkaya & $39^{\circ} 33^{\prime} 34.57^{\prime \prime} \mathrm{N}$ & $35^{\circ} 23^{\prime} 11.62^{\prime \prime} \mathrm{E}$ \\
\hline 20 & Yozgat-Central & $39^{\circ} 48^{\prime} 16^{\prime \prime} \mathrm{N}$ & $34^{\circ} 48^{\prime} 40^{\prime \prime} \mathrm{E}$ \\
\hline 21 & Yozgat-Boğazliyan & $39^{\circ} 19^{\prime} 55^{\prime \prime} \mathrm{N}$ & $35^{\circ} 08^{\prime} 35^{\prime \prime} \mathrm{E}$ \\
\hline 22 & Yozgat-Central & $39^{\circ} 49^{\prime} 26.25^{\prime \prime} \mathrm{N}$ & $34^{\circ} 51^{\prime} 58.77^{\prime \prime} \mathrm{E}$ \\
\hline 23 & Antalya-Akseki & $37^{\circ} 02^{\prime} 11.43^{\prime \prime} \mathrm{N}$ & $31^{\circ} 46^{\prime} 50.60^{\prime \prime} \mathrm{E}$ \\
\hline 24 & Yozgat-Central & $39^{\circ} 50^{\prime} 28.22^{\prime \prime} \mathrm{N}$ & $34^{\circ} 48^{\prime} 22.05^{\prime \prime} \mathrm{E}$ \\
\hline 25 & Amasya-Merzifon & $40^{\circ} 52^{\prime} 06.65^{\prime \prime} \mathrm{N}$ & $35^{\circ} 28^{\prime} 46.51^{\prime \prime} \mathrm{E}$ \\
\hline 26 & Yozgat-Sorgun & $39^{\circ} 50^{\prime} 44.86^{\prime \prime} \mathrm{N}$ & $35^{\circ} 65^{\prime} 44.68^{\prime \prime} \mathrm{E}$ \\
\hline 27 & Yozgat-Çandır & $39^{\circ} 14^{\prime} 47.68^{\prime \prime} \mathrm{N}$ & $35^{\circ} 32^{\prime} 07^{\prime \prime} \mathrm{E}$ \\
\hline
\end{tabular}


Table 2. Total bioactive compounds and radical scavenging activities of methanol extracts of roots of chicory, roasted fruits of turpentine tree, instant coffee, raw roasted coffee beans.

\begin{tabular}{|c|c|c|c|c|}
\hline Genotypes & $\begin{array}{c}\text { Total Flavonoid Contents } \\
(\mathrm{mg} \mathrm{QE} / \mathrm{g} \mathrm{DW})\end{array}$ & $\begin{array}{l}\text { Total Fenolic Contents } \\
\text { (mg GAE /g DW) }\end{array}$ & $\begin{array}{l}\text { ABTS Radical Cation } \\
\text { (\% inhibition) }\end{array}$ & $\begin{array}{l}\text { DPPH Radical } \\
\text { (\% inhibition) }\end{array}$ \\
\hline 1 & $0.396^{\mathrm{klma}}$ & $2.077^{\mathrm{j}-\mathrm{m}}$ & $33.65^{\text {efg }}$ & $8.437^{\mathrm{cd}}$ \\
\hline 2 & 0.570 hij & $1.873^{\mathrm{k}-\mathrm{p}}$ & $28.64 \mathrm{ij}$ & $7.787^{\mathrm{cd}}$ \\
\hline 3 & $0.636^{\mathrm{ghi}}$ & $2.423 \mathrm{hij}$ & $26.79^{\mathrm{jk}}$ & $7.607^{\mathrm{cd}}$ \\
\hline 4 & $0.413 \mathrm{kl}$ & $2.870 \mathrm{fg}$ & $24.14^{\mathrm{lm}}$ & 7.223 cde \\
\hline 5 & $0.673 \mathrm{fg}$ & $1.703^{1-p}$ & $20.83^{n}$ & $2.810^{\mathrm{gh}}$ \\
\hline 6 & $0.653 \mathrm{gh}$ & 3.203 ef & $25.79 \mathrm{klm}$ & $8.507^{\mathrm{cd}}$ \\
\hline 7 & $0.360^{\mathrm{k}-\mathrm{n}}$ & 1.567 nор & $24.99 \mathrm{klm}$ & $7.697 \mathrm{~cd}$ \\
\hline 8 & $0.336^{\mathrm{k}-\mathrm{n}}$ & 2.527 ghi & $13.36^{\mathrm{p}}$ & $8.327^{\mathrm{cd}}$ \\
\hline 9 & $0.513^{\mathrm{i}}$ & $1.860^{\mathrm{k}-\mathrm{p}}$ & $29.39^{\mathrm{i}}$ & $8.910^{c}$ \\
\hline 10 & $0.880^{\mathrm{e}}$ & $2.170^{\mathrm{ijk}}$ & $25.04 \mathrm{klm}$ & 5.290 ef \\
\hline 11 & $0.310^{\mathrm{m}}$ & $1.457 \mathrm{p}$ & $26.29 \mathrm{kl}$ & $7.200^{\text {cde }}$ \\
\hline 12 & $0.300^{n}$ & $1.550^{\mathrm{op}}$ & $38.61^{\mathrm{d}}$ & $8.170^{\mathrm{cd}}$ \\
\hline 13 & $1.063^{\mathrm{d}}$ & $3.533^{\mathrm{e}}$ & $33.30 \mathrm{fg}$ & $8.573^{\mathrm{cd}}$ \\
\hline 14 & $0.426^{\mathrm{k}}$ & $1.983^{\mathrm{j}-\mathrm{o}}$ & $20.43^{n}$ & $8.077^{\mathrm{cd}}$ \\
\hline 15 & $1.120^{\mathrm{d}}$ & $3.363^{\mathrm{e}}$ & $31.95^{\mathrm{gh}}$ & $8.417^{\mathrm{cd}}$ \\
\hline 16 & $0.383^{\mathrm{k}-\mathrm{n}}$ & $2.157 \mathrm{ijk}$ & 34.50 ef & nd \\
\hline 17 & $0.326^{\mathrm{lmn}}$ & $1.623^{\mathrm{m}-\mathrm{p}}$ & $29.34^{i}$ & $7.967^{\mathrm{cd}}$ \\
\hline 18 & $0.296^{\mathrm{n}}$ & $0.943^{\mathrm{q}}$ & $16.63^{\circ}$ & $3.823 \mathrm{fg}$ \\
\hline 19 & $0.616^{\text {ghi }}$ & $2.737^{\mathrm{gh}}$ & $35.77^{\mathrm{e}}$ & $1.620^{\mathrm{h}}$ \\
\hline 20 & $0.390^{\mathrm{k}-\mathrm{n}}$ & $2.137^{\mathrm{i}-\mathrm{k}}$ & $23.59^{\mathrm{m}}$ & $8.370^{\mathrm{cd}}$ \\
\hline 21 & $0.516^{\mathrm{j}}$ & $1.803^{\mathrm{k}-\mathrm{p}}$ & $24.79 \mathrm{klm}$ & $8.057^{\mathrm{cd}}$ \\
\hline 22 & $0.863^{\mathrm{e}}$ & $1.793^{\mathrm{k}-\mathrm{p}}$ & $24.94 \mathrm{klm}$ & nd \\
\hline 23 & $1.146^{\mathrm{d}}$ & $2.677^{\mathrm{gh}}$ & $26.69^{\mathrm{jk}}$ & $7.920^{\mathrm{cd}}$ \\
\hline 24 & $0.570^{\mathrm{hij}}$ & $1.433 \mathrm{p}$ & $23.84^{\mathrm{m}}$ & nd \\
\hline 25 & $0.526^{\mathrm{ij}}$ & $2.000^{j-n}$ & $30.04 \mathrm{hi}$ & $8.010^{\mathrm{cd}}$ \\
\hline 26 & $0.740^{\mathrm{f}}$ & $2.067^{j-n}$ & 33.95 efg & $6.680^{\mathrm{de}}$ \\
\hline 27 & $0.353^{\mathrm{k}-\mathrm{n}}$ & $1.423^{\mathrm{p}}$ & $26.64^{\mathrm{jk}}$ & $4.647 \mathrm{fg}$ \\
\hline RFT & $4.350^{\mathrm{a}}$ & $5.550^{c}$ & $88.63^{a}$ & $13.727^{b}$ \\
\hline IC & $1.163^{\mathrm{b}}$ & $13.860^{\mathrm{a}}$ & $69.80^{c}$ & $8.413^{\mathrm{cd}}$ \\
\hline $\mathrm{RCB}$ & 0.567 hij & $4.433^{\mathrm{d}}$ & $77.97^{b}$ & $21.917^{\mathrm{a}}$ \\
\hline $\mathrm{CB}$ & $1.443^{c}$ & $7.090^{b}$ & $76.26^{b}$ & $20.913^{\mathrm{a}}$ \\
\hline
\end{tabular}

In each column different letters indicate significant difference (P<0.01); nd: not determined; QE: Quercetin equivalents; GAE: Gallic acid equivalents; 1-27: Roots of chicory plants (Cichorium intybus) collected from different localities; RFT: Roasted fruits of turpentine tree (Pistacia terebinthus); IC: instant coffee; RCB: Roasted coffee beans; CB: Raw coffee beans.

According to previous studies, raw coffees beans have high polyphenol content, which is particularly rich in chlorogenic acid and related compound (Clifford 1999, Suzuki et al., 2002), reaching up to $14 \%$ (dry matter basis). However, it has been determined that processing, especially roasting modifies the phenolic composition of coffee, producing aroma, flavor and color compounds characteristics of coffee beverage significantly (Farah and Donangelo, 2006), and this shows similarity with the present study. Besides, total flavonoid content of roasted coffee beans was less than raw coffee beans' similar to the total phenolic content. On the other hand, it was assumed that roasted fruits extract of turpentine tree had the highest total flavonoid content (4.350 $\left.\mathrm{mg} \mathrm{QE} \mathrm{g}^{-1} \mathrm{DW}\right)$.

All the assessed extracts for DPPH activity were able to reduce the initial stable blue/purple DPPH radical to a yellow DPPH-H (Cavar et al., 2012, Khadhri et al., 2017). In the present study, the DPPH radical scavenging activity was changed based on the genotypes, coffee varieties, rawroasted coffee, and was listed here from high to low value: raw coffee beans $=$ roasted coffee beans $>$ roasted fruits of turpentine tree $>$ instant coffee $=$ roots of chicory. Unlike total phenolic and flavonoid contents, higher DPPH radical scavenging activities were obtained from raw and roasted coffee beans. The capability of different phenolic substances to scavenge various types of oxidationinitiating radicals has been reported, demonstrating different effects (Rive-Evans et al., 1996, Yen and Duh, 1994). For example; polyphenolic compounds have antioxidant activity in many studies (Okuda et al., 1994).

The ABTS free radical scavenging effects of all the tested samples of the plant were denoted in Table 2. The highest ABTS radical scavenging activity was found in extract of roasted fruits of turpentine tree $(88.63 \%)$, indicating more accumulation of flavonoids in this extract. However, the lowest was found in extracts of roots of chicory. Raw and roasted coffee beans $(76.26 \%, 77.97 \%$, respectively) were statistically placed in the same group and exhibited more activity than instant coffee (69.80\%).

The total phenolic and flavonoid content, DPPH and ABTS free radical scavenging activities of mix extracts of roasted coffee bean and root of chicory (20-80, 40-60, 8020 as $\%$ in $1 \mathrm{mg} / \mathrm{ml}$ extract) were given in Figure 1 . It was determined that the total phenolic contents, DPPH and ABTS activities in the mix extract increased with increasing ratio of roasted coffee bean, but, the total flavonoid contents decreased. This result was observed in all investigated genotypes of chicory $\left(6^{\text {th }}, 16^{\text {th }}\right.$ and $20^{\text {th }}$ genotypes). Specific synergistic effect was not observed in mix extracts. 
Table 3. Crude ash ratio and $\mathrm{P}, \mathrm{Ca}, \mathrm{Mg}(\%), \mathrm{Zn}, \mathrm{Fe}(\mathrm{ppm})$ content of chicory, roasted fruits of turpentine tree, instant coffee, raw roasted coffee beans

\begin{tabular}{|c|c|c|c|c|c|c|}
\hline Genotypes & Crude ash ratio & $\mathrm{P}$ content & Ca content & Mg content & Zn content & Fe content \\
\hline \begin{tabular}{l|}
1 \\
1
\end{tabular} & $4.75^{\mathrm{k}-\mathrm{n}}$ & $2.66^{\mathrm{f}-\mathrm{j}}$ & $0.18^{\mathrm{j}-\mathrm{n}}$ & $0.62 \mathrm{klm}$ & $13.40^{\mathrm{j}}$ & $19.93^{\mathrm{lmn}}$ \\
\hline 2 & $4.66^{1-0}$ & $2.44^{\mathrm{h}-\mathrm{k}}$ & $0.20^{\mathrm{h}-\mathrm{n}}$ & $0.65^{\mathrm{i}-\mathrm{m}}$ & $18.70^{e-h}$ & $27.92^{\mathrm{h}-\mathrm{k}}$ \\
\hline 3 & $5.54 \mathrm{gh}$ & $3.02^{\mathrm{c}-\mathrm{g}}$ & $0.27^{\mathrm{d}-\mathrm{j}}$ & $1.05 \mathrm{bc}$ & $19.51^{\text {e-h }}$ & $43.63^{\mathrm{e}}$ \\
\hline 4 & $4.53^{\mathrm{n}-\mathrm{q}}$ & $2.96^{\mathrm{d}-\mathrm{g}}$ & $0.24^{\mathrm{e}-\mathrm{m}}$ & $0.76^{e-j}$ & 16.14 hij & $26.84 \mathrm{ijk}$ \\
\hline 5 & $4.93^{\mathrm{j}-\mathrm{m}}$ & $3.08^{\mathrm{c}-\mathrm{f}}$ & $0.27^{\mathrm{d}-\mathrm{k}}$ & $0.90^{\mathrm{de}}$ & $20.13^{\text {efg }}$ & $37.49^{f}$ \\
\hline 6 & $4.49^{n-q}$ & $2.87^{\mathrm{d}-\mathrm{h}}$ & $0.20^{\mathrm{h}-\mathrm{n}}$ & $0.67^{\mathrm{h}-\mathrm{m}}$ & $14.95^{\mathrm{ij}}$ & $27.33^{\mathrm{h}-\mathrm{k}}$ \\
\hline 7 & $9.16^{b}$ & $2.61^{f-j}$ & $0.38^{b c}$ & $0.76^{\mathrm{e}-1}$ & $20.25^{\mathrm{efg}}$ & $52.95^{\mathrm{d}}$ \\
\hline 8 & $5.60^{\mathrm{gh}}$ & $1.96^{\mathrm{kl}}$ & $0.17^{\mathrm{k}-\mathrm{n}}$ & $0.62 \mathrm{klm}$ & $16.88^{g-j}$ & $29.20^{\mathrm{g}-\mathrm{j}}$ \\
\hline 9 & $5.10^{\mathrm{ijk}}$ & $2.53^{\mathrm{g}-\mathrm{j}}$ & $0.18^{\mathrm{j}-\mathrm{n}}$ & $0.81^{\mathrm{e}-\mathrm{i}}$ & $14.91^{\mathrm{ij}}$ & $34.70^{\mathrm{fg}}$ \\
\hline 10 & $7.33^{d}$ & $3.92^{a b}$ & $0.32^{\mathrm{c}-\mathrm{g}}$ & $1.20^{\mathrm{ab}}$ & $25.15^{b c}$ & $63.08^{c}$ \\
\hline 11 & $8.03^{c}$ & $3.02^{\mathrm{c}-\mathrm{g}}$ & $0.34^{\mathrm{cd}}$ & $1.13^{\mathrm{abc}}$ & $24.36^{\mathrm{bcd}}$ & $71.06^{b}$ \\
\hline 12 & $9.95^{\mathrm{a}}$ & $2.73^{\mathrm{f}-\mathrm{i}}$ & $0.43^{\mathrm{b}}$ & $1.21^{\mathrm{a}}$ & $25.05^{b c}$ & $92.52^{\mathrm{a}}$ \\
\hline 13 & $6.83^{\mathrm{e}}$ & 3.29 cde & $0.32^{\mathrm{c}-\mathrm{f}}$ & $1.17^{\mathrm{ab}}$ & $28.73^{\mathrm{a}}$ & $45.07^{\mathrm{e}}$ \\
\hline 14 & $6.15^{f}$ & $3.34^{\mathrm{cd}}$ & $0.33^{\text {cde }}$ & $1.14^{\mathrm{ab}}$ & $27.79^{a b}$ & $55.93^{\mathrm{d}}$ \\
\hline 15 & $4.58^{\mathrm{m}-\mathrm{p}}$ & $3.34^{\mathrm{cd}}$ & $0.27^{\mathrm{d}-\mathrm{k}}$ & $0.99^{\mathrm{cd}}$ & $22.11^{\mathrm{cde}}$ & $32.29^{f-i}$ \\
\hline 16 & $5.88^{\mathrm{fg}}$ & $2.23 \mathrm{ijk}$ & $0.23^{f-n}$ & $0.70^{\mathrm{g}-\mathrm{m}}$ & $21.96^{\mathrm{cde}}$ & $29.56^{\mathrm{g}-\mathrm{j}}$ \\
\hline 17 & $4.67^{1-o}$ & $2.22 \mathrm{ijk}$ & $0.14^{\mathrm{n}}$ & $0.60^{\mathrm{k}-\mathrm{n}}$ & $17.45^{\mathrm{f}-\mathrm{i}}$ & 13.39 орq \\
\hline 18 & $5.52^{\mathrm{h}}$ & $2.24 \mathrm{ijk}$ & $0.20^{\mathrm{h}-\mathrm{n}}$ & $0.63^{\mathrm{j}-\mathrm{m}}$ & $19.51^{\mathrm{e}-\mathrm{h}}$ & $33.42^{\text {fgh }}$ \\
\hline 19 & 4.95 ijk & $2.79 \mathrm{fgh}$ & $0.18^{i-n}$ & $0.79^{e-j}$ & $17.06^{\mathrm{ghi}}$ & $25.22^{\mathrm{jkl}}$ \\
\hline 20 & $7.41^{\mathrm{d}}$ & $2.65^{f-j}$ & $0.28^{\mathrm{d}-\mathrm{i}}$ & $0.86^{\text {def }}$ & $18.72^{\text {e-h }}$ & $54.27^{\mathrm{d}}$ \\
\hline 21 & $4.33^{\mathrm{o}-\mathrm{r}}$ & $3.06^{\mathrm{c}-\mathrm{f}}$ & $0.26^{\mathrm{d}-1}$ & $0.89 \mathrm{de}$ & $25.01^{b c}$ & $28.08^{\mathrm{h}-\mathrm{k}}$ \\
\hline 22 & $5.72^{\mathrm{gh}}$ & $2.82^{\mathrm{e}-\mathrm{h}}$ & $0.19^{\text {h-n }}$ & $0.61^{\mathrm{k}-\mathrm{n}}$ & $25.12^{b c}$ & $23.30^{\mathrm{j}-\mathrm{m}}$ \\
\hline 23 & $4.19 \mathrm{qr}$ & $2.41^{\mathrm{h}-\mathrm{k}}$ & $0.19^{\text {h-n }}$ & $0.70^{\mathrm{f}-\mathrm{m}}$ & $17.88^{\mathrm{f}-\mathrm{i}}$ & $25.58^{\mathrm{jkl}}$ \\
\hline 24 & $5.13 \mathrm{ij}$ & $2.44^{\mathrm{h}-\mathrm{k}}$ & $0.16^{\mathrm{lmn}}$ & $0.60^{\mathrm{lmn}}$ & $20.08^{e f g}$ & $22.07 \mathrm{klm}$ \\
\hline 25 & $5.42 \mathrm{hi}$ & $1.64 \mathrm{~lm}$ & $0.16^{\mathrm{lmn}}$ & $0.58 \mathrm{mn}$ & 16.05 hij & $24.911^{\mathrm{jkl}}$ \\
\hline 26 & $4.78^{j-n}$ & $4.30^{\mathrm{a}}$ & $0.24^{\mathrm{e}-\mathrm{m}}$ & $0.84^{\mathrm{d}-\mathrm{g}}$ & $28.89^{\mathrm{a}}$ & $24.74^{\mathrm{jkl}}$ \\
\hline 27 & $3.85^{t}$ & $2.28^{\mathrm{ijk}}$ & $0.17^{\text {k-n }}$ & $0.73^{\mathrm{f}-\mathrm{m}}$ & $21.86^{\text {cde }}$ & $15.48^{\text {nор }}$ \\
\hline RFT & $4.85^{\mathrm{j}-\mathrm{n}}$ & $0.69^{n}$ & $0.54^{\mathrm{a}}$ & $0.83^{\mathrm{e}-\mathrm{i}}$ & $6.95^{1}$ & $18.13^{\mathrm{mno}}$ \\
\hline IC & $4.34^{\mathrm{o}-\mathrm{r}}$ & $0.55^{\mathrm{n}}$ & $0.40^{\mathrm{bc}}$ & $0.64^{\mathrm{j}-\mathrm{ml}}$ & $6.16^{1}$ & $8.63 \mathrm{qr}$ \\
\hline RCB & $4.23 \mathrm{pqr}$ & $0.37^{\mathrm{n}}$ & $0.28^{\mathrm{d}-\mathrm{h}}$ & 0.47 no & $6.00^{1}$ & $7.45^{\mathrm{s}}$ \\
\hline $\mathrm{CB}$ & $2.61^{\mathrm{u}}$ & $1.22^{\mathrm{m}}$ & $0.61^{\mathrm{a}}$ & $0.35^{\circ}$ & $10.02^{j}$ & $23.23^{\mathrm{j}-\mathrm{m}}$ \\
\hline
\end{tabular}

In each column different letters indicate significant difference (P<0.01); nd: not determined; QE: Quercetin equivalents; GAE: Gallic acid equivalents; 1-27: Roots of chicory plants (Cichorium intybus) collected from different localities; RFT: Roasted fruits of turpentine tree (Pistacia terebinthus); IC: instant coffee; RCB: Roasted coffee beans; CB: Raw coffee beans.

Crude ash ratio and mineral element contents $(\mathrm{P}, \mathrm{Ca}$, $\mathrm{Mg}, \mathrm{Zn}, \mathrm{Fe}, \mathrm{Mn}, \mathrm{S}, \mathrm{B}, \mathrm{Cr}, \mathrm{Co}$ and $\mathrm{Mn}$ ) in the chicory genotypes and coffees are presented in Table 3 and 4 . The highest crude ash ratios $(9.95 \%)$ was found in $12^{\text {th }}$ genotype, while the lowest (2.61) was found in raw coffee beans. Kim et al. (1978) reported the crude ash ratio of chicory root ranged between $3.0-4.2 \%$.

The content of $\mathrm{P}, \mathrm{Ca}$ and $\mathrm{Mg}$ ranged between 1.22$4.30 \%, 0.14-0.61 \%$ and $0.35-1.21 \%$ respectively. Durrani et al. (2010) reported that potassium is an essential element for normal body growth, while Calcium is an important constituent of bones and teeth and it is actively involved in the regulation of nerve and muscle functions. It was reported that $\mathrm{Ca}$ and $\mathrm{Mg}$ content in instant coffee were $0.78 \%$ and $0.21 \%$ respectively (Demir et al., 2015). Yiteyal and Tilahun (2017) reported that the average amount of calcium and phosphorus required for infants is 230-187.5 $\mathrm{mg} /$ day, for children is $850-480 \mathrm{mg} /$ day and for other age groups is $1150-975 \mathrm{mg} / \mathrm{day}$. The present study shows that the $\mathrm{Ca}$ and $\mathrm{P}$ contents of chicory roots are sufficient. Previous researchers indicated that coffees made from other plants have less $\mathrm{Mg}$ than chicory (Suseela et al., 2001; Chaves et al., 2012; Samsonowicz et al., 2019).

The highest $\mathrm{Fe}$ and $\mathrm{Mn}$ contents were obtained from the $12^{\text {th }}$ genotype (92.52 and $61.86 \mathrm{ppm}$, respectively), while the lowest were found in roasted coffee beans ( 7.45 and $4.38 \mathrm{ppm}$, respectively). Kim et al. (1978) reported the $\mathrm{Fe}$ content of chicory root ranged between 90-120 ppm. Yiteyal and Tilahun (2017) reported that Mn is necessary for the development and growth of organisms, and 0.6-2.6 $\mathrm{mg}$ of Mn should be consumed daily.

The content of $\mathrm{B}, \mathrm{Cr}, \mathrm{Co}$ and Mo ranged between 2.2314.81 ppm, 0.15-3.99 ppm, 0.22-12.37 ppm and 4.28-20.47 ppm, respectively. Comparing the results obtained in this study with the data in the literature (Table 4), chicory root can be said to have higher cobalt content than others plant. (Suseela et al., 2001; Chaves et al., 2012; Samsonowicz et al., 2019).

The biplot graphic analyses of the 27 chicory genotypes and coffee varieties are shown in Figure 2. According to the antioxidant properties and mineral contents that sum of values in PCA $1(49.65 \%)$ and PCA $2(17.98 \%)$ were $67.63 \%$. In the present study, the coffee varieties had higher values compared to the chicory genotypes in terms of the antioxidant properties, while chicory genotypes had higher mineral content compared to the coffee varieties. Besides, genotypes of $3^{\text {th }}, 5^{\text {th }}, 7^{\text {th }}, 10^{\text {th }}, 11^{\text {th }}, 12^{\text {th }}, 13^{\text {th }}, 14^{\text {th }}$, $15^{\text {th }}, 20^{\text {th }}, 21^{\text {th }}$ and $26^{\text {th }}$ had higher mineral contents compared to the other chicory genotypes. 
Table 4. Mn, S, B, Cr, Co and Mo content of chicory, roasted fruits of turpentine tree, instant coffee, raw roasted coffee beans (ppm)

\begin{tabular}{|c|c|c|c|c|c|c|}
\hline Genotypes & Mn content & S content & B content & Cr content & Co content & Mo content \\
\hline 1 & $21.19 \mathrm{hij}$ & $745.7^{\mathrm{i}-1}$ & $10.501^{\mathrm{f}-\mathrm{k}}$ & $0.158^{\circ}$ & $2.281^{\mathrm{hi}}$ & 11.558 \\
\hline 2 & $30.08^{f}$ & $692.8^{\mathrm{i}-1}$ & $9.881^{\mathrm{g}-1}$ & $1.127 \mathrm{mn}$ & $1.286^{\mathrm{ij}}$ & 15.465 \\
\hline 3 & $36.73 \mathrm{de}$ & $1596.4^{\text {a }}$ & $13.400 \mathrm{abc}$ & $1.650^{\mathrm{g}-\mathrm{j}}$ & 5.069 ef & 20.473 \\
\hline 4 & $18.88^{\mathrm{ijk}}$ & $700.5^{\mathrm{i}-1}$ & $12.502^{\mathrm{b}-\mathrm{e}}$ & $1.638^{\mathrm{g}-\mathrm{k}}$ & $3.168^{\text {gh }}$ & 11.730 \\
\hline 5 & $29.34 \mathrm{fg}$ & $919.5^{f-i}$ & $12.962 \mathrm{bcd}$ & $1.569^{\mathrm{h}-1}$ & $4.320^{\mathrm{fg}}$ & 12.154 \\
\hline 6 & $18.944^{\mathrm{ijk}}$ & $875.7^{g-j}$ & $10.230^{\mathrm{f}-\mathrm{k}}$ & $2.071 \mathrm{de}$ & $2.463^{\mathrm{hi}}$ & 11.209 \\
\hline 7 & $36.56^{\mathrm{de}}$ & $633.8 \mathrm{kl}$ & $9.317^{\mathrm{i}-1}$ & $1.360^{\mathrm{im}}$ & $7.476^{\mathrm{cd}}$ & 14.256 \\
\hline 8 & $21.32 \mathrm{hij}$ & $564.2^{1}$ & $8.960^{\mathrm{jkl}}$ & $2.324^{\mathrm{cd}}$ & $7.680^{\mathrm{cd}}$ & 11.412 \\
\hline 9 & $21.45^{\text {hij }}$ & $640.4^{\mathrm{kl}}$ & $9.438^{h-1}$ & $1.547^{\mathrm{h}-\mathrm{l}}$ & $4.199^{\mathrm{fg}}$ & 8.630 \\
\hline 10 & $46.80^{\mathrm{c}}$ & $1199.4^{\text {cde }}$ & $11.599^{\mathrm{c}-\mathrm{g}}$ & $1.716^{\text {fgh }}$ & $8.835^{b c}$ & 14.901 \\
\hline 11 & $52.38^{\mathrm{b}}$ & $1003.7^{e-h}$ & $11.954^{b-f}$ & $2.560^{\mathrm{c}}$ & $9.787^{\mathrm{b}}$ & 12.876 \\
\hline 12 & $61.86^{\mathrm{a}}$ & $1258.0^{\mathrm{cd}}$ & $11.979^{b-f}$ & $3.579^{\mathrm{b}}$ & $12.379^{\mathrm{a}}$ & 12.534 \\
\hline 13 & $49.67^{b c}$ & $1347.5^{\mathrm{bc}}$ & $14.818^{\mathrm{a}}$ & $1.334^{\mathrm{j}-\mathrm{m}}$ & $7.332^{\mathrm{cd}}$ & 16.745 \\
\hline 14 & $51.52^{b c}$ & $1082.8^{\mathrm{d}-\mathrm{g}}$ & $13.187^{\mathrm{a}-\mathrm{d}}$ & $1.951^{\mathrm{efg}}$ & $8.007^{\mathrm{cd}}$ & 16.863 \\
\hline 15 & $29.38^{\mathrm{fg}}$ & $1088.0^{\mathrm{d}-\mathrm{g}}$ & $13.097^{\mathrm{a}-\mathrm{d}}$ & $2.407^{\mathrm{c}}$ & $4.748^{\mathrm{fg}}$ & 13.509 \\
\hline 16 & $27.56^{\mathrm{fg}}$ & $717.6^{\mathrm{i}-1}$ & $10.713^{e-j}$ & $1.689^{f-i}$ & $6.939^{d}$ & 12.575 \\
\hline 17 & $21.63^{\mathrm{hij}}$ & $758.9^{\mathrm{i}-1}$ & $9.386^{\mathrm{i}-1}$ & $1.960^{\mathrm{efg}}$ & $3.171^{\text {gh }}$ & 10.031 \\
\hline 18 & 31.71 ef & $794.1^{\mathrm{h}-1}$ & $9.677^{\text {h-1 }}$ & $0.848^{\circ}$ & $6.536^{\mathrm{de}}$ & 10.379 \\
\hline 19 & $30.43^{\mathrm{f}}$ & $736.5^{\mathrm{i}-1}$ & $9.297^{\mathrm{i}-1}$ & $1.593^{\mathrm{h}-\mathrm{k}}$ & $6.951^{\mathrm{d}}$ & 13.669 \\
\hline 20 & $38.64^{\mathrm{d}}$ & $821.5^{\mathrm{h}-\mathrm{k}}$ & $11.360^{\mathrm{d}-\mathrm{h}}$ & $1.253^{\mathrm{lm}}$ & $7.101^{\mathrm{d}}$ & 11.355 \\
\hline 21 & $24.26^{\text {ghi }}$ & 1095.2 def & $13.510^{\mathrm{ab}}$ & $3.995^{\mathrm{a}}$ & 3.462 fgh & 15.160 \\
\hline 22 & 36.68 de & $736.6^{\mathrm{i}-\mathrm{l}}$ & $11.071^{\mathrm{e}-\mathrm{i}}$ & $1.585^{\mathrm{h}-\mathrm{k}}$ & $4.564^{\mathrm{fg}}$ & 13.643 \\
\hline 23 & 24.33 ghi & $573.0^{1}$ & $10.112^{\mathrm{f}-\mathrm{k}}$ & $1.051^{\mathrm{mn}}$ & $4.412^{\mathrm{fg}}$ & 11.037 \\
\hline 24 & $26.49^{\text {fgh }}$ & $748.9^{\mathrm{i}-1}$ & $9.484^{h-1}$ & $1.520^{\mathrm{h}-\mathrm{l}}$ & $6.312^{\mathrm{de}}$ & 49.388 \\
\hline 25 & $16.76^{\mathrm{jkl}}$ & $749.5^{\mathrm{i}-1}$ & $8.556^{\mathrm{kl}}$ & $1.935^{\mathrm{efg}}$ & $3.297 \mathrm{gh}$ & 9.332 \\
\hline 26 & $53.57^{\mathrm{b}}$ & $1466.9^{a b}$ & $12.385^{\mathrm{b}-\mathrm{e}}$ & $1.496^{\mathrm{h}-\mathrm{l}}$ & $3.680^{\text {fgh }}$ & 17.549 \\
\hline 27 & $16.18^{\mathrm{jkl}}$ & $887.2^{\mathrm{f}-\mathrm{j}}$ & $9.543^{h-1}$ & $1.128 \mathrm{mn}$ & $2.383^{\mathrm{hi}}$ & 12.083 \\
\hline RFT & $13.72 \mathrm{klm}$ & $863.9^{\mathrm{h}-\mathrm{k}}$ & 6.697 m & $0.871^{\mathrm{n}}$ & $0.669^{\mathrm{j}}$ & 6.374 \\
\hline IC & $12.27^{\mathrm{lm}}$ & $766.0^{\mathrm{i}-1}$ & $4.370^{n}$ & $0.204^{\circ}$ & $0.653^{\mathrm{j}}$ & 6.653 \\
\hline $\mathrm{RCB}$ & $4.38^{\mathrm{n}}$ & $857.8^{\mathrm{h}-\mathrm{k}}$ & $2.232^{\circ}$ & $0.242^{\circ}$ & $0.425^{\mathrm{j}}$ & 5.465 \\
\hline $\mathrm{CB}$ & $8.65^{\mathrm{mn}}$ & $719.4^{\mathrm{i}-1}$ & $4.444^{\mathrm{n}}$ & $0.274^{\circ}$ & $0.221^{\mathrm{j}}$ & 4.284 \\
\hline
\end{tabular}

In each column different letters indicate significant difference ( $\mathrm{P}<0.01)$; nd: not determined; QE: Quercetin equivalents; GAE: Gallic acid equivalents; 1-27: Roots of chicory plants (Cichorium intybus) collected from different localities; RFT: Roasted fruits of turpentine tree (Pistacia terebinthus); IC: instant coffee; RCB: Roasted coffee beans; CB: Raw coffee beans.

\section{Conclusion}

In the present study, the $6^{\text {th }}, 13^{\text {th }} .15^{\text {th }}$ genotypes for total phenolic content and $13^{\text {th }}, 15^{\text {th }}, 23^{\text {th }}$ genotypes for total flavonoid content had the highest total bioactive content with regards to the genotypes of $C$. intybus. Likewise, the $1^{\text {th }}, 6^{\text {th }}$, $13^{\text {th }}, 15^{\text {th }}$ genotypes for DPPH, and the $1^{\text {st }}, 12^{\text {th }}, 16^{\text {th }}, 19^{\text {th }}$ genotypes for ABTS had the highest radical scavenging activity. Moreover, the $3^{\text {th }}, 5^{\text {th }}, 7^{\text {th }}, 10^{\text {th }}, 11^{\text {th }}, 12^{\text {th }}, 13^{\text {th }}, 14^{\text {th }}$, $15^{\text {th }}, 20^{\text {th }}, 21^{\text {th }}$ and $26^{\text {th }}$ genotypes had the highest value in terms of mineral contents. Chicory roots showed low antioxidant activity, but, higher mineral content compared to the other tested coffee varieties. As a result, the $13^{\text {th }}$ and the $15^{\text {th }}$ genotypes can be recommended as a coffee additive in terms of antioxidant properties and mineral contents.

\section{Acknowledgements}

This text has been proofread and edited by the Department of Foreign Languages, Bilecik Şeyh Edebali University.

\section{References}

Ahmad B, Siddiqui AB, Alam T, Alam SA. 2002. Components from seed of Cichorium intybus Linn. Indian Journal of Chemistry, 41B (12): 2701-5.
Anonymous 2019. Available from: https://mgm.gov. tr/?il=Yozgat. [Accessed 28 February 2019].

Arvouet-Grand A, Vennat B, Pourrat A, Legret P. 1994. Standardisation d un extrait de propolis et identification des principaux constituants. Journal de pharmacie de Belgique, 49: 462-468.

Cavar S, Vidic D, Maksimovic M. 2012. Volatile constituents, phenolic compounds, antioxidant activity of Calamintha glandulosa (Req.) Bentham. Journal of the Science of Food and Agriculture, 93: 1758-1764.

Chávez, DWH, Ascheri JLR, Carvalho CWP, Godoy RLO, Pacheco S. 2017. Sorghum and roasted coffee blends as a novel extruded product: Bioactive compounds and antioxidant capacity. Journal of Functional Foods, 29: 93103.

Clifford MN. 1999. Chlorogenic acids and other cinnamates: nature, occurrence and dietary burden. Journal of the Science of Food and Agriculture, 79: 362-372.

Demir F, Selvi N, Seyhun Kıpcak A, Özgül DO, Piskin MB, Derun MD. 2015. The Investigation of the Element Contents in the Turkish Coffees. Celal Bayar University Journal of Science, 11 (3): 423-428.

Denev P, Petkova N, Ivanov I, Sirakov B, Vrancheva R, Pavlov A. 2014. Determination of Biologically Active Substances in Taproot of Common Chicory (Cichorium intybus 1.). Scientific Bulletin. Series F. Biotechnologies, 18: 124-129. 
Duarte MP, Laires A, Gaspar J, Oliveira JS, Rueff J. 2000. Genotoxicity of Instant Coffee and of some Phenolic Compounds Present in Coffee Upon Nitrosation. Teratogenesis, Carcinogenesis, and Mutagenesis, 20: 241-249.

Durrani Y, Ayub M, Muhammad A, Ali A. 2010. Pysicochemical response of apple pulp to chemical preservatives and antioxidant during storage. Journal of Food Safety, 12: 20-28.

Ghamarian A, M Abdollahi, X Su, A Amiri, A Ahadi, and A Nowrouzi. 2012. Effect of chicory seed extract on glucose tolerance test (GTT) and metabolic profile in early and latestage diabetic rats. Daru, 20 (1): 56.

Farah A, and CM Donangelo. 2006. Phenolic compounds in coffee. Brazilian Journal of Plant Physiology, 18 (1): 23-36.

Franck A. 2002. Technological functionality of inulin and oligofructose. The British Journal of Nutrition, 87: 287-291.

Gezer K, Duru ME, Kıvrak I, Türkoğlu A, Mercan N, Türkoğlu H, Gülcan S. 2006. Free-radical Scavenging Capacity and Antimicrobial Activity of Wild Edible Mushroom of Turkey. African journal of Biyotechnology, 5 (20): 1924-1928.

Hasanloo T, Sepehrifar R, Hajimehdipoor H. 2011. Levels of phenolic compounds and their eff ects on antioxidant capacity of wild Vaccinium arctostaphylos L. (Qare-Qat) collected from diff erent regions of Iran. Turkish Journal Biology, 35: 371-377.

Heimler D, Isolani L, Vignolini P, Romani A. 2009. Polyphenol content and antiradical activity of Cichorium intybus L. from biodynamic and conventional farming. Food Chemistry, 114: $765-770$.

Jurgonski A, Juskiewicz J, Zdunczyk Z, Król B. 2012. Caffeoylquinic acid-rich extract from chicory seeds improves glycemia, atherogenic index, and antioxidant status in rats. Nutrition, 28 (3): 300-6.

Khadhri A, Bouali I, Belkhir S, Mokded R, Smiti S, Falé P, Eduarda M, Araújo M, Serralheiro MLM. 2017. In vitro digestion, antioxidant and antiacetylcholinesterase activities of two species of Ruta: Ruta chalepensis and Ruta montana, Pharmaceutical Biology, 55 (1): 101-107.

Kier AM, Mes THM, Van der Meijden R, Bachmann K. 1999. Morphologically defined Cichorium (Asteraceae) species reflect lineages based on chloroplast and nuclear (ITS) DNA data. Systematic Botany, 24: 645-659.

Kim TY, Yoon YJ, Lee KW. 1978. Studies of Constituents of the Chicory Root. Korean Journal Food Science Technology, 10 (2): 258-262.

Kaskoos RA. 2012. Anti-diabetic activity of Cichorium intybus seeds on STZ-induced diabetic rats. International Research Journal of Pharmacy, 3 (5): 161-4.
Lavelli V. 2008. Antioxidant activity of minerally processed red chicory (Cichorium intybus L.) evaluated in xanthine oxidase-myeloperoxidase and diaphorase-catalyzed reactions. Journal of Agricultural and Food Chemistry, 56 (7): 194-200.

Miller NJ, Rice-Evans C, Devies MJ, Gopinathan V, Milner A. 1993. A novel method for measuring antioxidant capacity and its application to monitoring the antioxidant status in premature neonates. Clinical Science, 84: 407-412.

Nandagopal S, Ranjitha Kumari BD. 2007. Phytochemical and Antibacterial Studies of Chicory (Cichorium intybus L.) - A Multipurpose Medicinal Plant. Advances in Biological Research, 1 (1-2): 17-21.

Okuda T, Yoshida T, Hatano T. 1994. Food phytochemicals for cancer prevention II. In: Chemistry and antioxidative effects of phenolic compounds from licorice, tea and compositae and labiateae herbs, ed. Ho CT, Osawa T, Huang MT, Rosen RT. American Chemical Society, Washington, pp. 132-143.

Re R, Pellegrini N, Proteggente A, Pannala A, Yang M, RiceEvans C. 1999. Antioxidant activity applying an improved ABTS radical cation decolorization assay. Free Radical Biology \& Medicine - Journal, 26: 1231-1237.

Rive-Evans CA, Miller NJ, Paganga G. 1996. Structureantioxidant activity relationships of flavonoids and phenolic acids. Free Radicals in Biology and Medicine, 20: 933-956.

Samsonowicz M, Regulska E, Karpowicz D, Lesniewska B. 2019. Antioxidant properties of coffee substitutes rich in polyphenols and minerals. Food Chemistry, 278: 101-109.

Schumacher E, Vigh E, Molnár V, Kenyeres P, Fehér G, Késmárky G. 2011. Thrombosis preventive potential of chicory coffee consumption: Phytotherapy Research, 25 (5): 744-8.

Singleton VL, Orthofer R, Lamuela-Raventos RM. 1999. Analysis of total phenols and other oxidation substrates and antioxidants by means of Folin-Ciocalteu reagent. Methods Enzymol, 299: 152-178.

Suseela B, Bhalke S, Kumar V. 2001. Daily intake of trace metals through coffee consumption in India. Food Additives and Contaminants, 18(2): 115-120.

Suzuki A, Kagawa D, Ochiai R, Tokimitsu I, Saito I. 2002. Green coffee bean extract and its metabolites have a hypotensive effect in spontaneously hypertensive rats. Hypertension Research, 25: 99-107.

Yen GC, Duh PD. 1994. Scavenging effect of methanolic extracts of peanut hulls on free-radical and active oxygen species. Journal of Agricultural and Food Chemistry, 42: 629-632.

Yiteyal AA, Tilahun RD. 2017. Nutritional Value of Coffee Made from a Mixture of Coffee Leaf and Spices Used in Ethiopia. Journal of Nutrition \& Food Sciences, 7 (4): 618. 